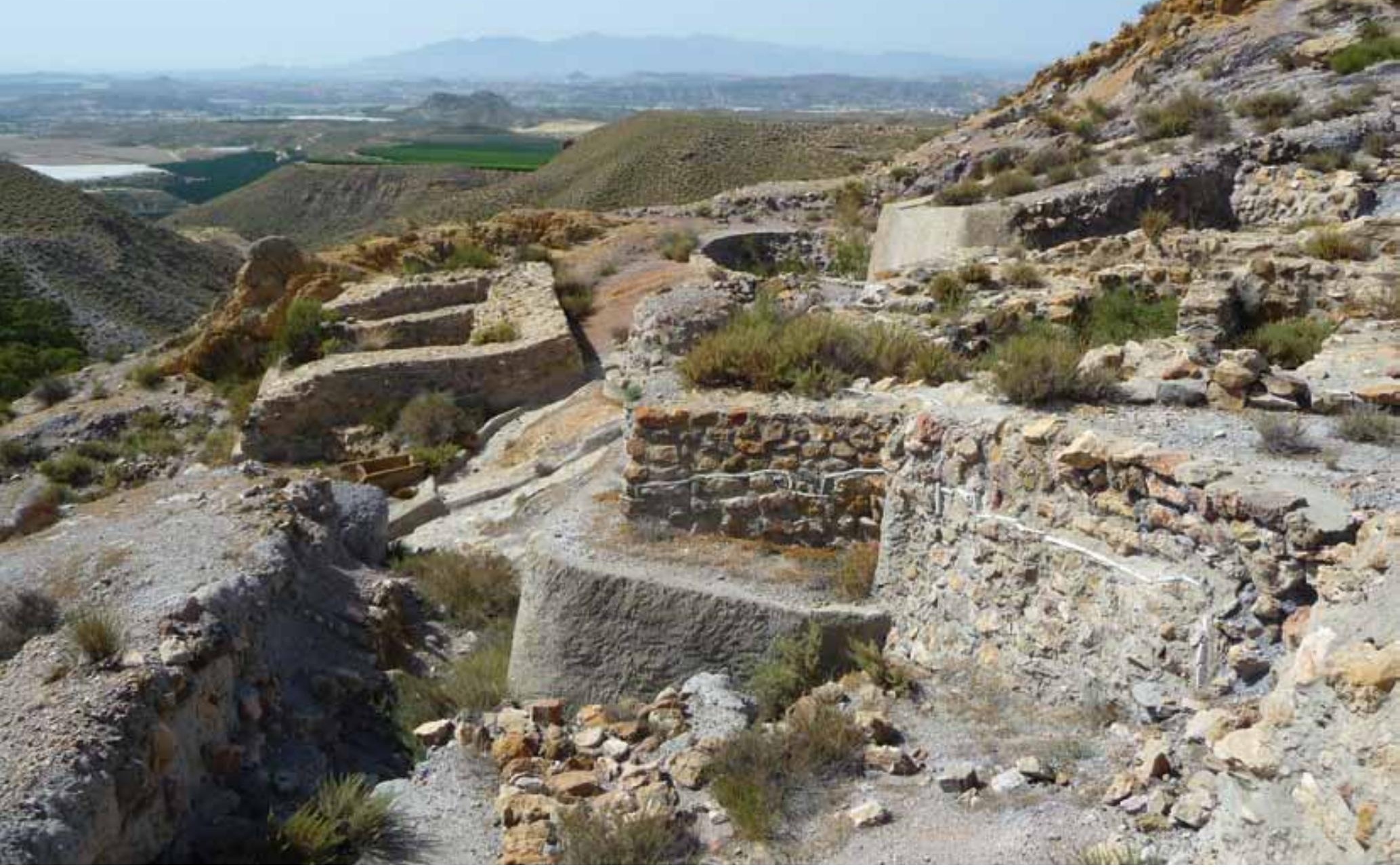

Fuente Álamo. Foto: Vicente Camarasa Domínguez 


\section{Los paisajes siretianos: ciento veinte años de intervención sobre el patrimonio arqueológico prehistórico}

Margarita Sánchez Romero, prehistoriadora, Directora General de Bienes Culturales, Consejería de Cultura de la Junta de Andalucia
Hay lugares en el mundo en los que el vínculo que se establece entre los restos materiales de las sociedades del pasado y el medio natural en el que se encuentran es tan intenso que crean un paisaje único, cargado de significados, que constituye una identidad particular e irrepetible. La simple mención de hitos geográficos como la sierra de Atapuerca o el valle del Neander evoca de inmediato la presencia de restos arqueológicos. Algo muy similar ocurre con el territorio definido por las cuencas de los ríos Almanzora, Antas y Aguas, donde se concentran una considerable cantidad de yacimientos arqueológicos cuyo estudio ha sido fundamental para caracterizar culturas muy significativas de la prehistoria reciente europea. Estos lugares configuran un paisaje privilegiado, un lugar en el que sentir la emoción del estar, del contemplar, del comprender, del conocer in situ algunos de los lugares arqueológicos más significativos de las edades del Cobre y Bronce europeas.

Pero además de compartir un espacio y un tiempo, estos yacimientos comparten el entusiasmo y la dedicación de unos hermanos belgas, Luis y Enrique Siret, ingenieros que llegaron al sureste de la Peninsula Ibérica para acometer la construcción de infraestructuras relacionadas con la explotación minera. La espectacularidad de los hallazgos que realizaron y el desarrollo de una minuciosa y casi "icónica" documentación dan coherencia y significado a estos lugares que, desde entonces, conocemos como paisajes siretianos. El propio Antonio Arribas, catedrático de prehistoria de la Universidad de Granada, reconoció y asumió la categoria de "siretólogo" para aquellos que sienten la misma pasión que Luis Siret por los sitios y paisajes prehistóricos de Almería (ARRIBAS PALAU, 1994).

Entre los años 1881 y 1887 los hermanos Siret excavaron yacimientos como los de La Pernera, El Argar, La Gerundia, Cabezo del Moro, Cruz de Antas, Qurenima, Lugarico Viejo, Fuente Bermeja o El Garcel en Antas; Almizaraque, Fuente Álamo, Tres Cabezos, El oficio, Barranco Hondo, Zájara, Campos, Herrerías, Villaricos, Loma de la Encantada o Puerto Blanco en Cuevas del Almanzora; Caldero de Mojácar, Cuartillas y Raja de Ortega en Mojácar; Gatas en Turre o Tres Palacés en Zurgena. Todas estas intervenciones aparecen en la obra Las primeras edades del metal en el sudeste de España que vio la luz, primero, en Amberes en 1887, y luego, en 1890, en Barcelona, y que sigue siendo referente en cualquier biblioteca especializada en arqueología (SIRET; SIRET, 1890).

Los trabajos de investigación realizados por Enrique y Luis Siret desde 1880, y continuados desde 1886 por Luis Siret y su capataz Pedro Flores hasta la muerte del primero en 1934, se convierten en referentes teóricos y metodológicos en un debate que dura ya más de cien años. Estos yacimientos pasan a ser el escenario de los cambios que se han ido produciendo en la investigación arqueológica en nuestro pais, desde finales deI XIX hasta la actualidad, y situaron a las cuencas del Almanzora, Antas y Aguas en el mapa del mundo arqueológico. Gracias a estos trabajos de Luis Siret, los conocieron prominentes figuras de la arqueología del momento: Pierre Paris, Raymond Lantier, Manuel Gómez-Moreno, Cayetano de Mergelina, Pedro Bosch Gimpera, Luis Pericot, Vera Leisner, Georg Leisner, Adolf Schulten, Henri Breuil, Juan Cabré o Émile Cartailhac pasaron en distintos momentos por Herrerías donde el ingeniero tenía su casa (LÓPEZ CASTRO, 2004).

Sin duda alguna, una de sus aportaciones más reconocidas, debido probablemente a su formación como ingenieros, se produce en el ámbito de la metodología. Las intervenciones de los hermanos Siret se caracterizan por la rigurosidad en la documentación y por una importante documentación topográfica que completan con planimetrías y alzados de las construcciones; en algunas ocasiones sitúan los distintos materiales que aparecen con relación a las estructuras documentadas y se preocupan por la reconstrucción de los procesos constructivos y la génesis de los depósitos (ARANDA JIMÉNEZ, 2009).

Pero aunque la metodología suele ser lo más reseñado de entre las aportaciones de los Siret, quizá sea en el debate teórico donde podemos ver reflejada la importancia real de estos territorios para la explicación de las sociedades de la prehistoria reciente. El dinamismo en el cambio cultural y su relación con el proceso 
de transformación social ya fue advertido por los hermanos Siret desde el primer momento $y$, gracias a sus investigaciones, el sureste de la Península Ibérica se ha convertido desde finales del siglo XIX en un escenario privilegiado para el estudio del proceso de jerarquización social, siendo utilizado por varias generaciones de investigadores para articular propuestas de cambio cultural (ARANDA JIMÉNEZ, 2011).

Son tres las etapas que han sido articuladas para la explicación de los cambios teóricos e ideológicos (ARANDA JIMÉNEZ, 2011). Una primera que abarcaría desde las investigaciones de los hermanos Siret hasta los años 70 del siglo XX, en la que el modelo colonial fue aceptado de manera unánime; la segunda, desde los años 70 a principios de los 90, caracterizada por las energías puestas en la investigación de campo y por el planteamiento de novedosas teorias de corte autoctonista realizadas básicamente desde perspectivas funcionalistas y materialistas; y una tercera, que llegaría hasta nuestros dias, en la que el debate sobre los diferentes modelos interpretativos se ha situado en el ámbito de la contrastación empírica, para lo que la información arqueológica obtenida en la etapa anterior se ha convertido en el elemento de referencia imprescindible.

En la etapa inaugurada por L. Siret, las propuestas difusionistas eran las utilizadas para tratar de explicar la variabilidad cultural del sureste peninsular de manera que cualquier cambio cultural se vinculaba a la llegada de poblaciones del mediterráneo oriental u otros ámbitos europeos. Estas hipótesis prevalecieron en el discurso arqueológico durante varias décadas y sólo a partir de finales de la década de los 70 y durante los 80 comienza a desarrollarse en nuestro pais la crítica al historicismo y al modelo difusionista. Buen ejemplo de ello son los trabajos de corte materialista de Antonio Gilman (1985) y Vicente Lull (1983) o las investigaciones de Robert Chapman o Clay Mathers realizadas desde perspectivas funcionalistas que potenciaron enormemente el debate sobre los procesos de cambio cultural. De esta forma, se pasa a un nuevo escenario en donde las comunidades del sureste son consideradas como protagonistas de su propio proceso histórico.

A lo largo de más de un siglo de investigación, el registro arqueológico de estos yacimientos se ha utilizado para contestar preguntas sobre jerarquización social, organización política, procesos de agregación poblacional, comportamientos culturales frente a escenarios medioambientales diversos, gestión de bienes fundamentales como el agua u organización de la producción tanto de productos agricolas y ganaderos como otro tipo de bienes (objetos metálicos, cerámica). Finalmente, junto al debate sobre el apoyo empírico de las propuestas planteadas en los 70 y 80 , en estos últimos años han comenzado a desarrollarse nuevas perspectivas que ponen el acento en cómo las diferentes comunidades definen, negocian y modifican sus identidades sociales (género, edad y condición social) a través de diferentes prácticas sociales, rituales y de complejos procesos de resistencia, hibridación, asimilación o emulación (ARANDA JIMÉNEZ, 2011).
A la interesante historia del desarrollo teórico-metodológico de la arqueología prehistórica podemos sumar también la actuación de la administración cultural sobre estos lugares y conocer el recorrido conceptual sobre el patrimonio arqueológico. En 1984 se producen las trasferencias en materia de cultura desde el Estado a la Junta de Andalucía y se impone una preocupación real por impulsar todas las acciones de la tutela del patrimonio, es decir, la protección, la conservación, la investigación y la difusión del patrimonio arqueológico andaluz.

En materia de arqueología, durante una primera etapa los esfuerzos se dedicaron a la realización de catálogos e inventarios de yacimientos para su protección y a la investigación arqueológica como única acción posible en la que basar la tutela. Es el momento del denominado modelo andaluz de arqueología que creó la figura del proyecto de investigación para planificar las intervenciones, fomentar la interdisciplinariedad e impulsar la publicación de los resultados como elemento básico en la intervención sobre yacimientos arqueológicos. Se desarrollan los proyectos La edad del Cobre en la cuenca del río Almanzora (Almería), dirigido por María Dolores Cámalich Massieu y Dimas Martín Socas, de la Universidad de La Laguna; el Proyecto Gatas. Sociedad y economía en el sudeste de España c. 2500-800 a.n.e., dirigido por Vicente Lull, Pedro Castro y Robert W. Chapman, los dos primeros de la Universidad de Barcelona; o Investigación arqueológica en los poblados de la edad del Bronce de Fuente Álamo y El Argar, dirigido por Hermanfrid Schubart y Volker Pingel, del Instituto Arqueológico Alemán. En años posteriores Catalina Martínez Padilla, de la Universidad de Almería, dirigió el Proyecto Alto Almanzora. Estudio del proceso histórico durante la Prehistoria y la Antigüedad en la cuenca del Alto Almanzora, Almería, y Vicente Lull, de la Universidad Autónoma de Barcelona, continuó con los trabajos en Turre con el proyecto Gatas 2.

Estas investigaciones dan lugar a una serie de publicaciones monográficas como El territorio almeriense desde los inicios de la producción hasta fines de la Antigüedad: un modelo, la depresión de Vera y cuenca del Río Almanzora, por María Dolores Cámalich Massieu y Dimas Martín Socas (1998); Proyecto Gatas. 2, La dinámica arqueológica de la ocupación prehistórica, por Pedro V. Castro Martínez y otros (1999); o Fuente Álamo: las excavaciones arqueológicas 1977-1991 en el poblado de la Edad del Bronce, por Hermanfrid Schubart, Volker Pincel y Oswaldo Arteaga (2000).

La nómina de intervenciones en yacimientos prehistóricos en el territorio que analizamos se ha ido ampliando a lo largo de los años y se ha llevado a cabo una importante labor de protección; en la actualidad existen dos decenas de yacimientos prehistóricos protegidos en esta área y hay otros tantos expedientes en elaboración. En cuanto a las intervenciones en conservación, se han llevado a cabo trabajos de consolidación, vallado y señalización y puesta en valor de yacimientos como Fuente Álamo, acometidos por el Instituto Arqueológico Alemán, o Villaricos, en el que 


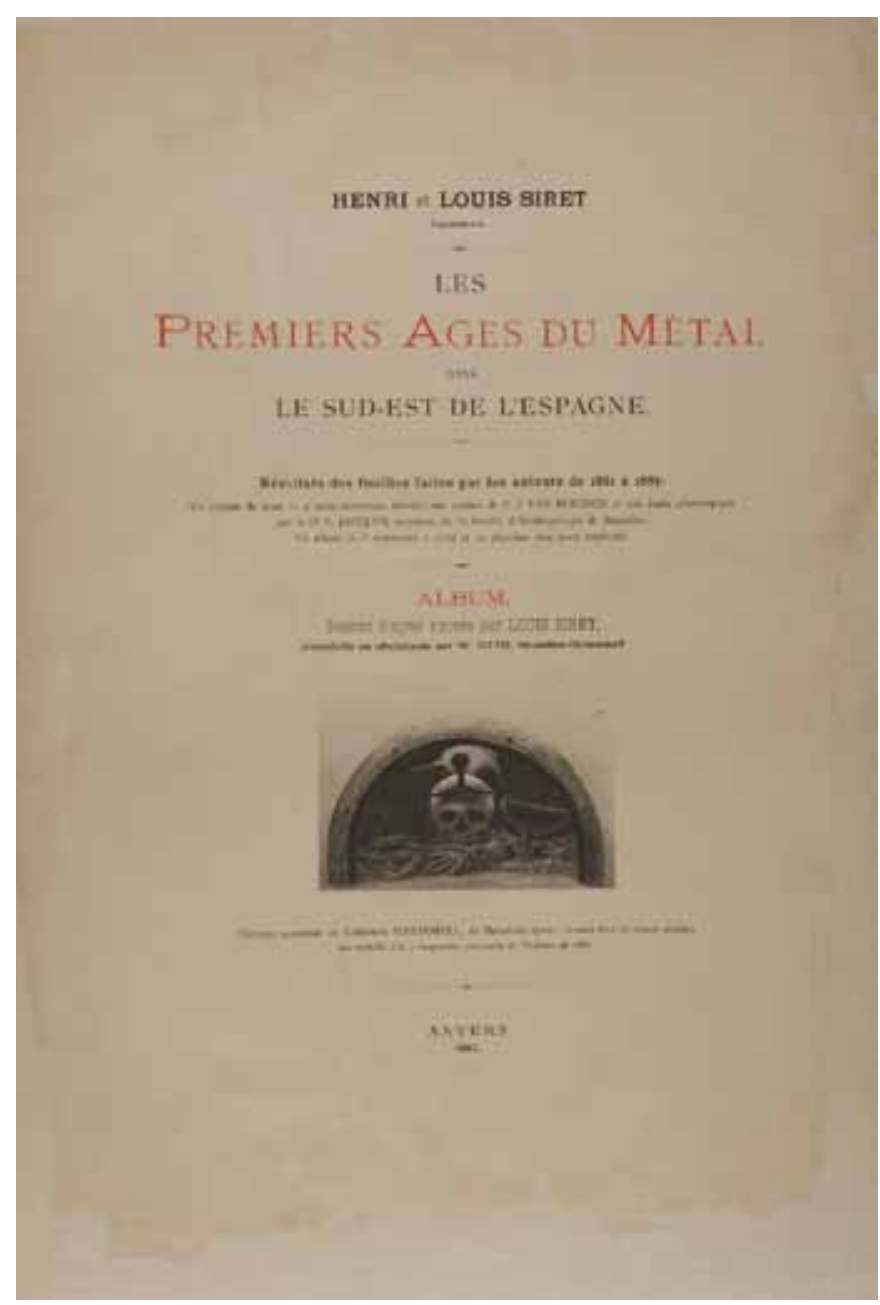

Portada de la publicación de los hermanos Siret titulada Les prermiers ages du metal dans le sud-est de l'Espagne de 1887

se han realizado diversas obras de consolidación de los hipogeos, de cerramiento y señalización de la necrópolis y de construcción de un centro de recepción de visitantes. También se acometió un importante programa de restauración de la casa de Luis Siret entre 1993 y 1998 (SUÁREZ MÁROUEZ, 2011).

La riqueza arqueológica y el potencial de estos territorios debe hacernos reflexionar sobre nuestra forma de intervenir en ellos empleando los procesos clave de la tutela: la protección, la conservación y el uso de estos bienes patrimoniales de carácter arqueológico. Las tareas relativas a la protección y a la conservación tienen un largo arraigo en la administración cultural andaluza, aunque siempre sean susceptibles de mejorar; sin embargo, los nuevos retos que plantea la sociedad del siglo XXI reclaman del patrimonio arqueológico que ejerza los papeles que le confiere ser tanto un recurso para la cultura como un capital económico. La primera de las funciones está relacionada con la construcción de identidades sociales, con valores simbólicos, con el servicio a la educación y a la ciencia, con el estímulo de la conciencia ciudadana o con los valores sensitivos y de disfrute; como capital económico, el patrimonio está relacionado con la inserción en el sistema productivo, incluyendo la generación de rentas e incluso de empleo y, particularmente, con la inclusión en el tejido local a través de la participación en el desarrollo

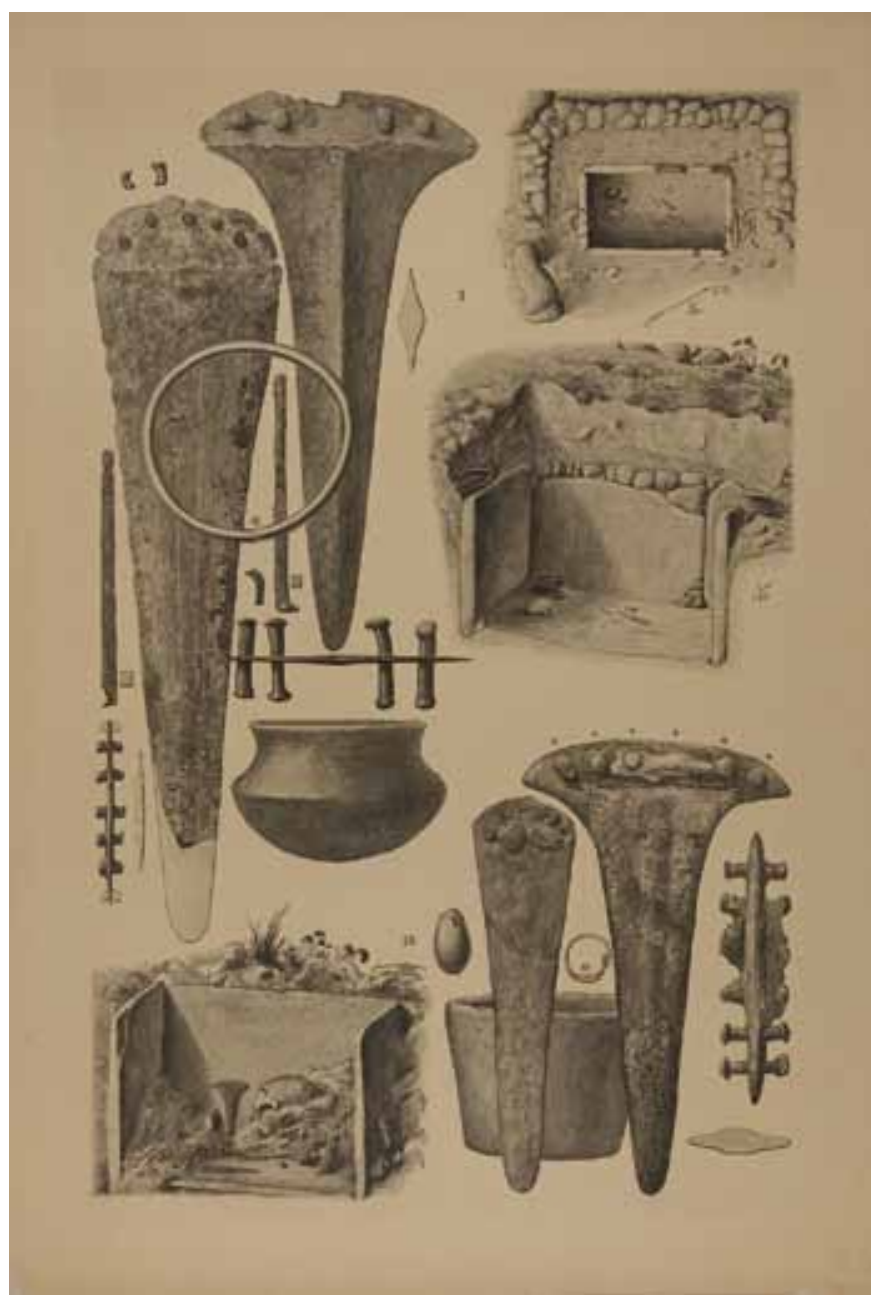

Planos y ajuares de las sepulturas 1 y 18 de la necrópolis de Fuente Álamo, incluidos en la misma publicación

regional (SÁNCHEZ ROMERO; RODRÍGUEZ DE GUZMÁN; HERNÁNDEZ DE LA OBRA, 2011).

Pero para hacer más eficiente este trabajo es necesario saber cuál es la situación de los lugares siretianos en los distintos procesos de tutela, y ya se trabaja en la redacción de un diagnóstico inicial del estado de protección, conservación, investigación, del conocimiento por parte de la ciudadanía y de sus posibles usos para, a partir de ese análisis, planificar nuestras actuaciones a través de un Plan Director, un documento estratégico que contenga las pautas para organizar, impulsar y orientar las actuaciones que se han de llevar a cabo durante los próximos años, estableciendo de forma encadenada y coherente entre sí la misión y visión institucional y una serie de objetivos, estrategias y líneas de acción que las desarrollen.

En este camino debemos además tener en cuenta que la tutela de los bienes culturales demanda la interdisciplinariedad, el aporte de recursos especializados, el apoyo tecnológico, la utilización de servicios, etc. que no siempre son propios de la misión de la administración cultural y que deben ser obtenidos desde agentes externos. Recibir el apoyo complementario desde estos agentes externos para complementar el trabajo administrativo de gestión -proceso de fuera a dentro- $y_{1}$ por 


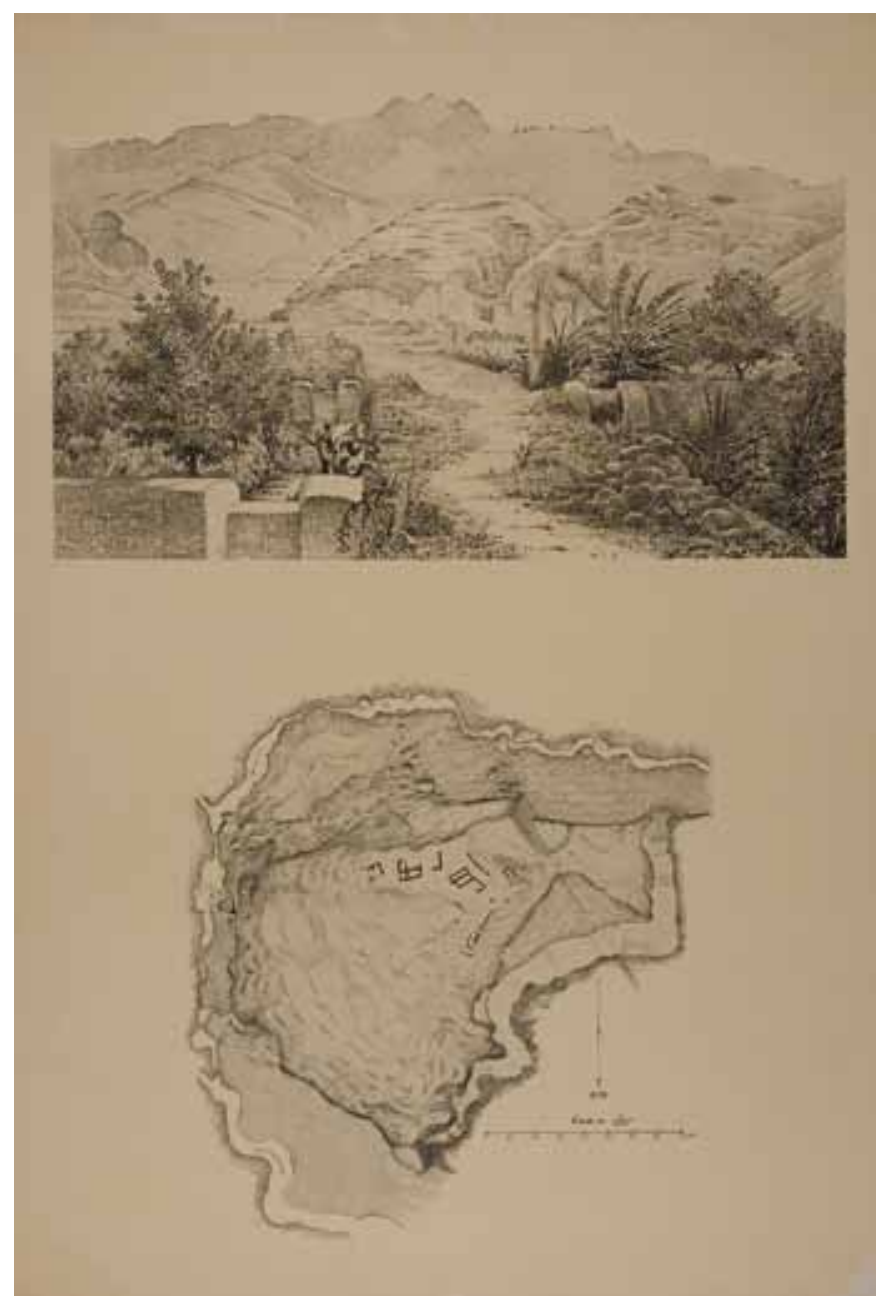

Vista y plano del yacimiento de Gatas, incluidos en la misma publicación

otra parte, ofrecer un servicio a la sociedad -proceso de dentro a fuera- son las dos funciones que se deben buscar con la cooperación entre la administración y los agentes externos. Se necesitará por tanto de la cooperación y el acuerdo entre las instituciones en el territorio, de la intervención de los agentes sobre el terreno (por ejemplo, los grupos de desarrollo rural) $y_{\text {, }}$ particularmente, de la participación en la gestión de la iniciativa empresarial de las industrias culturales, una estrategia de acción que debe ser planteada y con ella lograr la superación de un cierto recelo hacia la participación de estos agentes y la apertura de nuevas puertas a la gestión del patrimonio (SÁNCHEZ ROMERO; RODRÍGUEZ DE GUZMÁN; HERNÁNDEZ DE LA OBRA, 2011).

Ciento veinte años después, Siret, los "siretólogos" y los paisajes siretianos deben consolidarse como una unidad de referencia en la tutela del patrimonio arqueológico prehistórico; durante todo este tiempo hemos vinculado emocionalmente nuestras responsabilidades sobre el patrimonio con los trabajos realizados por Siret. En junio de 1984 se celebró en Cuevas del Almanzora (Almería) un congreso homenaje a la memoria del ingeniero en el que, además de reconocerlo como precursor de la investigación prehistórica en Andalucia, se analizó la situación de la arqueología prehistórica y protohistórica a
La espectacularidad de los hallazgos

de los hermanos Siret y el desarrollo de una minuciosa documentación, dan coherencia y significado a los "paisajes siretianos"

mediados de los años 80 en nuestra comunidad autónoma (AA. W., 1986). Veinticinco años más tarde, entre julio de 2009 y septiembre de 2010 se organizó el Memorial Luis Siret, que recorrió Almería, Cuevas del Almanzora, Águilas y finalmente Antequera, lugar de celebración del I Congreso de Prehistoria de Andalucía que, bajo el epigrafe La tutela del patrimonio prehistórico, permitió la revisión de lo sucedido en la arqueología prehistórica y protohistórica durante los años de administración autonómica en Andalucía, y que inaugura una serie de encuentros bienales en los que discutir aspectos teóricos y metodológicos en este materia.

Sin proponérselo, Siret diseñó una identidad arqueológica y paisajistica de las cuencas del Almanzora, el Antas y el Aguas que se ha convertido en un referente de excepcional atractivo y con múltiples posibilidades de disfrute. Nuestra tarea debe ser crear las condiciones necesarias para que eso suceda.

\section{Bibliografía}

AA. W. (1986) Homenaje a Luis Siret (1934-1984). Sevilla: Consejeria de Cultura, Junta de Andalucia, 1986

ARANDA JIMÉNEZ, G. (2009) Luis Siret. En DÍAZ-ANDREU, M.; MORA, G.; CORTADELLA, J. (coord.) Diccionario Histórico de la Arqueología en España. Madrid: Marcial Pons, 2009

ARANDA JIMÉNEZ, G. (2011) Nuevos actores para viejos escenarios. La sociedad argárica. Memorial Luis Siret. I Congreso de Prehistoria de Andalucia. La tutela del patrimonio prehistórico. Sevilla: Consejería de Cultura, Junta de Andalucía (en prensa)

ARRIBAS PALAU, A. (1994) Presentación. En Orientales y Occidentales en España en los tiempos prehistóricos. Luis Siret [1907]. Almería: Arráez Editores, 1994, pp. 9-16 (Colección Siret de Arqueologia)

GILMAN, A.; THORNES, J. B. (1985) Land-use and Prehistory in south-east of Spain. Londres: George Allen y Unwin, 1985

LÓPEZ CASTRO, J. L. (2004) Luis Siret y los inicios de la Arqueología en el Sureste de España. Mus-A: Revista de los museos de Andalucía, n. ${ }^{\circ}$, 2004, pp. 168-175

LULL, V. (1983) La cultura del argar. Un modelo para el estudio de las formaciones sociales prehistóricas. Barcelona: Crítica, 1983

\section{SÁNCHEZ ROMERO, M.; RODRÍGUEZ DE GUZMÁN, S.; HERNÁNDEZ DE}

LA OBRA, J. (2011) Sociedad y patrimonio: políticas públicas. En $8^{\circ}$ Seminario Arqueología y Enseñanza (31 de marzo al 2 de abril de 2011). Creando redes del pasado al futuro: patrimonio histórico y sociedad civil. Centre d'Estudis del Patrimoni Arqueològic de la Prehistòria, Universitat Autònoma de Barcelona (en prensa)

SIRET, H.; SIRET, L. (1890) Las primeras edades del metal en el sudeste de España. Barcelona,1890

SUÁREZ MÁROUEZ, A. (2011) La dificultad de conservación del patrimonio arqueológico. Memorial Luis Siret. I Congreso de Prehistoria de Andalucía. La tutela del patrimonio prehistórico. Sevilla: Consejeria de Cultura, Junta de Andalucia (en prensa) 